\title{
Vivax malaria and bacteraemia: a prospective study in Kolkata, India
}

\author{
Sujit Kumar Bhattacharya ${ }^{1}$, Dipika Sur ${ }^{2}$, Shanta Dutta ${ }^{2}$, Suman Kanungo ${ }^{2}$, R Leon Ochiai ${ }^{3}$, Deok Ryun Kim³, \\ Nicholas M Anstey ${ }^{4}$, Lorenz von Seidlein ${ }^{4}$ and Jacqueline Deen ${ }^{4 *}$
}

\begin{abstract}
Background: Falciparum malaria increases the risk for bacteraemia, whereas the relationship between vivax malaria and bacteraemia is not clear. Data from a prospective fever surveillance study in Kolkata, India were reanalysed for the potential association between Plasmodium vivax malaria and bacteraemia.

Methods: Patients of all ages presenting with fever of three days or more to a project health outpost were invited to participate. A blood film and blood culture was performed on presentation. Treatment and referral were provided according to national guidelines. The case fraction and incidence of malaria, bacteraemia, and co-infection were calculated.

Results: 3,371 participants were enrolled during a one-year study period, of whom 93/3,371 (2.8\%) had malaria (89/93 [95.7\%] Plasmodium vivax) and 256 (7.6\%) bacteraemia. There were 154 malaria, 423 bacteraemia and 10 P. vivax-bacteremia coinfection episodes per 100,000/year. Among the malaria-bacteraemia co-infections, all were vivax malaria and 5/6 (83\%) bacteria isolated were Gram-negative (one S. Typhi, one S. Paratyphi A, three other Gram-negative). Bacteraemia occurred in 6/89 (6.7\% [95\%Cl: 3.1-13.9\%]) of $P$. vivax cases versus 250/3,278 (7.6\% [95\% Cl: 6.7-8.6\%]) without Plasmodium infection ( $p=0.76)$.
\end{abstract}

Conclusions: While an increased risk was not demonstrated, concomitant bacteraemia occurs frequently in vivax malaria in an area with a high background incidence of bacteraemia, and should be considered in cases of vivax malaria with severe manifestations.

Keywords: Bacteraemia, Falciparum malaria, Vivax malaria

\section{Background}

The association between Plasmodium falciparum infection and bacteraemia is well documented [1]. A report from Nigeria suggested the association between invasive salmonellosis and falciparum malaria in children [2]. It was then shown that young Gambian children with invasive non-typhoidal Salmonella infection were more anaemic and more likely to have evidence of recent malaria than were children of the same age with other forms of septicaemia [3]. Studies in Kenya showed that an acute episode of falciparum malaria predisposes to bacteraemia and that the latter contributes to the clinical picture of severe malaria [4]. The association between non-typhoidal

\footnotetext{
* Correspondence: deen.jacqueline@gmail.com

${ }^{4}$ Menzies School of Health Research and Charles Darwin University, Rocklands Drive, Casuarina, NT 0810, Australia

Full list of author information is available at the end of the article
}

Salmonella bacteraemia and severe anaemia has also been found in Malawi [5,6]. A recent study in Kenya has provided the strongest evidence to date of $P$. falciparum infection increasing the risk for bacteraemia, particularly for Gram-negative invasive infections [7]. Strategies that control falciparum malaria reduce child mortality to a greater extent than can be attributed to malaria alone $[8,9]$, likely through its mitigation of bacterial disease.

In contrast to falciparum malaria, the relationship between vivax malaria and bacteraemia has been less explored. The rate of concomitant bacteraemia in severe Plasmodium vivax malaria is not known, nor the extent to which concomitant bacterial infections contributes to the manifestations of severe vivax disease. Although there are reports of uncomplicated and severe vivax malaria with concurrent bacteraemia [10-12], recent series of severe vivax malaria cases with sepsis-like syndromes, including

\section{Biomed Central}

(c) 2013 Bhattacharya et al.; licensee BioMed Central Ltd. This is an Open Access article distributed under the terms of the Creative Commons Attribution License (http://creativecommons.org/licenses/by/2.0), which permits unrestricted use, distribution, and reproduction in any medium, provided the original work is properly cited. 
shock and multi-organ dysfunction, have not included systematic collection of pre-antibiotic blood cultures [13-15]. Importantly, there are increasing reports of $P$. vivax with severe clinical syndromes [16]. Data from a prospective fever surveillance study was reanalysed to assess the frequency of concomitant bacteraemia in $P$. vivax-infected patients presenting for primary care treatment of fever and the possible association between these two conditions.

\section{Methods}

\section{Study population and procedures}

The study population and procedures have been previously described in detail [17]. Briefly, the site was in Kolkata, India and consisted of wards 29 and 30, which are legally registered urban slum areas (bustees). The area is densely populated with narrow streets, little space between houses, intermittent piped water supply and inadequate sanitation facilities. A 2004 study census recorded a population of 60,452 , of whom 15,980 were two months to 14 years of age [17]. Prospective fever surveillance was conducted from January-December 2004 in preparation for a large typhoid fever vaccine effectiveness trial [18]. Five project health outposts set-up in the study area were open daily from 08:00-20:00 hours. Two project health outposts in the city's infectious diseases hospital and children's referral hospital were open 24 hours a day. Each outpost, operated by a physician and five assistants, offered free treatment according to national guidelines or referral with free transport to hospital when required. From the study population, all patients with fever for three days or more, irrespective of age, were invited to participate.

From each participant, a drop of blood was used for a thin blood film and stained with Leishman's stain. At least 100 high-power thin film microscopic fields were examined to exclude the diagnosis of malaria. Plasmodium species were identified on the thin film and confirmed by a senior malariologist. Approximately 5-8 $\mathrm{ml}$ of blood from adults and 3-5 $\mathrm{ml}$ from children were collected and used to inoculate BACTEC-Plus Aerobic ${ }^{\circ}$ and BACTEC-Peds Plus $^{\oplus}$ culture vials (Becton Dickinson), respectively. Inoculated Bactec bottles were incubated at $37^{\circ} \mathrm{C}$ for seven days with subcultures made on MacConkey agar (Difco) during the 1st, 2nd, 4th, and 7th days of incubation. Colonies detected on the plates were checked by Gram staining and other biochemical tests following standard procedures. Identification of Salmonella enterica serotype Typhi and Paratyphi strains was confirmed by slide and tube agglutination using polyvalent and monovalent factor antisera (Becton Dickinson) for $\mathrm{O}$ and $\mathrm{H}$ antigens. Salmonella isolates were preserved in glycerol stock at $-70^{\circ} \mathrm{C}$, and their identities verified at a reference laboratory (University of Oxford-Wellcome Trust Clinical Research Unit, Ho Chi Minh City, Vietnam). Other Gram-negative and Gram-positive bacteria were not identified further.

\section{Data management, definitions and analysis}

Standard case report forms were used to record medical history, physical examination findings, laboratory results and clinical management. Data were double-entered into custom-made data entry programs (FoxPro, Microsoft Corp) including error, range and consistency check programs.

Fever was defined as history of a rise in body temperature as recalled by the patient/caregiver or presence of axillary temperature $\geq 37.5^{\circ} \mathrm{C}$ on presentation. Only one blood culture was performed (on enrolment). Bacterial isolates were classified as Gram-negative (including Salmonellae) or Gram-positive. Malaria was defined as fever with the presence of asexual malaria parasites on the blood film, classified as P. falciparum, P. vivax, Plasmodium malariae or Plasmodium ovale. Co-infection was defined as the presence of bacteraemia and malaria.

The one-year incidence (per 100,000 population) of fever episodes, malaria, bacteraemia and co-infections was estimated using the number of such cases residing in the referral population as the numerator and the age-specific population from a study census as the denominator. The incidence of $P$. vivax malaria and typhoid fever have been previously reported [17], but not of other bacteraemia and co-infections. The potential association between the two binary variables, $P$. vivax parasitaemia and bacteraemia on presentation for treatment, was assessed using the Chi square test. Data analysis was performed using Stata software, version 11.0. Statistical significance was designated at $\mathrm{p}$ value $<0.05$.

\section{Ethics}

Written informed consent was obtained from each patient or his/her parent or guardian prior to participation. The Health Ministry Screening Committee of the Government of India, the Institutional Review Board of the International Vaccine Institute (Seoul, Korea) and the Secretariat Committee for Research Involving Human Subjects, World Health Organization (Geneva, Switzerland) approved the study. The confidentiality of information collected from study participants, including those derived from clinical specimens was ensured during and after the conduct of the study. All participant data were computerized using password protection and all hard copy records were kept at a secure place and available only to authorized study personnel for research purposes.

\section{Results}

During the one-year study period, 3,371 (1,234 who were 2 months to 14 years of age) were enrolled and included in the analysis (Table 1). Malaria parasites were detected in the blood of 93/3,371 (2.8\%) participants. Of the malaria cases, 89/93 (95.7\%) were P. vivax, 2 (2.2\%) P. falciparum, one (1.1\%) P. malariae and one (1.1\%) unspecified. Bacteraemia was detected in $256 / 3,371$ (7.6\%) participants. Of the 
Table 1 Number (incidence per 100,000 population) of malaria, bacteraemia and co-infections

\begin{tabular}{lll}
\hline & All ages & $\begin{array}{l}\text { Children } \\
\mathbf{2} \text { months } \\
\text { to } \mathbf{1 4} \text { years }\end{array}$ \\
\hline Population denominator & 60,452 & 15,980 \\
No (\%) participants enrolled & $3,371(5.6)$ & $1,234(7.7)$ \\
Mean age of participants & 24.7 & 7.7 \\
No (\%) female participants & $1,641(48.7)$ & $591(47.9)$ \\
Number (incidence per 100,000 population) of: & \\
Malaria & $93(154)$ & $21(131)$ \\
Bacteraemia & $256(423)$ & $114(713)$ \\
Gram-positive bacteraemia & $32(53)$ & $4(25)$ \\
Gram-negative bacteraemia & $223(369)$ & $109(682)$ \\
Typhoid fever & $95(157)$ & $57(357)$ \\
Other Salmonellosis & $64(106)$ & $31(194)$ \\
(63 S. Paratyphi A and 1 other) & & \\
Number (incidence per 100,000 population) of co-infections: \\
Malaria \& bacteraemia & $6(10)$ & $2(13)$ \\
Falciparum malaria \& bacteraemia & $0(0)$ & $0(0)$ \\
Vivax malaria \& bacteraemia & $6(10)$ & $2(13)$ \\
Malaria \& Gram-positive bacteraemia & $1(2)$ & $0(0)$ \\
Malaria \& Gram-negative bacteraemia & $5(8)$ & $2(13)$ \\
Malaria \& typhoid fever & $1(2)$ & $1(6)$ \\
Malaria \& paratyphoid fever & $1(2)$ & $0(0)$ \\
\hline
\end{tabular}

256 bacterial isolates, 223 (87.1\%) were Gram-negative, including $S$. Typhi (95 or $42.6 \%$ ), S. Paratyphi A (63 or $28.3 \%$ ) and other Salmonellae (1 or $0.4 \%$ ). Of the 89 vivax malaria cases 6 (6.7\% [95\%CI: 3.1-13.9\%]) had concomitant bacteraemia but similarly, 250/3278 (7.6\% [95\% CI: 6.7-8.6\%]) of the non-malarious cases were also bacteraemia $(\mathrm{p}=0.76)$.

There were 154 malaria and 423 bacteraemia episodes per 100,000 population (131 and 713 in children) per year (Table 1). Malaria and bacteraemia co-infections occurred at 10 per 100,000 population (13 in children) per year. Among the malaria-bacteraemia co-infections, all were vivax malaria and 5/6 (83\%) bacteria isolated were Gram-negative (one $S$. Typhi, one $S$. Paratyphi A, three other Gram-negative). There was one vivax malaria and two typhoid fever cases who were hospitalized. None of the patients with concomitant malaria and bacteraemia were hospitalized. There were no deaths among the enrolled participants.

\section{Discussion}

In this Indian study site, a high proportion (6.7\%) of vivax malaria cases presenting for treatment had concomitant bacteraemia. With $7.6 \%$ of non-malarious cases also bacteraemic, it could not be demonstrated that $P$. vivax infection increases the risk of bacteraemia. Nevertheless, in areas like
Kolkata, with a high incidence of both vivax malaria and bacteraemia, co-infections are common.

The high frequency of $P$. vivax-bacteraemia co-infection in this Indian site is notable because a number of series from across this region have reported $P$. vivax infection with severe sepsis-like syndromes [14,16]. Bacterial co-infections could contribute to severe sepsis-like presentations reported with $P$. vivax parasitaemia [10-16]. In falciparum malaria, bacteraemia, malnutrition and HIV infection are biologically associated with severe disease and an increased risk of death, rather than just being alternative diagnoses in coincidentally parasitized patients [19]. Similar relationships are plausible in vivax malaria where severe disease may arise from $P$. vivax infection, bacterial infection, or an additive or synergistic combination of both, often with other comorbidities [16]. Hypnozoite activation by significant systemic illness leading to vivax relapse has also been proposed [20].

The study has several limitations. The confidence interval for the $6.7 \%$ frequency of $P$. vivax-bacteraemia co-infection is wide (3.1-13.9\%); an increased risk of bacteraemia from $P$. vivax cannot be excluded. Considering the insensitivity of blood cultures and widespread community antibiotic usage, the frequency of bacteraemia and bacteremia- $P$. vivax coinfection may be higher. Second, since both malaria and bacteraemia were diagnosed and treated at the same time (on presentation), only the rates of concurrent vivax malaria and bacteraemia and a potential association between both could be assessed and not whether the former predisposes to the latter or vice versa. And third, since majority of cases were captured in the community and treated early, cases with severe manifestations were uncommon with only three patients hospitalized. Despite these limitations, this study provides the largest and best available population-based dataset on the relationship between $P$. vivax infection and bacteraemia.

The relationship between $P$. vivax malaria and bacteraemia is important to understand. P. vivax is a major cause of morbidity and mortality and is estimated to comprise around $40 \%$ of the world's malaria burden [21]. As current malaria control and elimination programmes disproportionately reduce the incidence of $P$. falciparum, the global fraction of malaria due to $P$. vivax is expected to rise. Whether $P$. vivax increases the risk for bacteraemia, the extent to which concurrent bacterial infection contributes to manifestations and outcomes of severe disease in $P$. vivax infection and whether systemic bacterial infections increase vivax relapses all require further elucidation. Multi-country, hospital-based studies with systematic, quality-assured pre-antibiotic blood and other sterile-site cultures in vivax malaria patients, especially those with severe manifestations, are needed, as well as follow-up studies of bacteraemic patients to evaluate the risk of vivax relapse. 


\section{Conclusions}

In a region with a high risk of bacterial infection, concomitant bacteraemia in $P$. vivax infection is common. Severe sepsis-like syndromes occurring in association with $P$. vivax infection require blood cultures and consideration of empiric broad-spectrum antimicrobial treatment. Further studies are needed to understand the interaction between vivax malaria and bacteraemia.

\section{Competing interests}

The authors declare that they have no competing interests.

\section{Authors' contributions}

Design and implementation of the field studies: SKB, DS, SK, RLO, JD and LVS. Laboratory supervision: SD. Conception of the current analysis: NA. Design of current analysis: LvS and JD. Statistical analysis: DRK. Wrote the first draft: NA, LVS and JD. Participated in the revision of the manuscript: SKB, DS, SD, RLO, DRK, NA, LVS, and JD. All authors read and approved the final manuscript.

\section{Acknowledgements}

We are grateful to the patients and their parents who made this work possible. We acknowledge all technical staff and research assistants who were involved in clinical and laboratory data collection.

This work was supported by the Bill and Melinda Gates Foundation, administered by the International Vaccine Institute, Seoul, Korea. NA is supported by an Australian Government National Health and Medical Research Council Practitioner Fellowship. LvS is supported by the Asia Pacific Malaria Elimination Network funded by the Australian Agency for International Development. The funding sources had no role in the writing of the manuscript and the decision to submit it for publication.

\section{Author details}

${ }^{1}$ Society for Applied Studies, Kolkata, India. ${ }^{2}$ National Institute of Cholera and Enteric Diseases, Kolkata, India. International Vaccine Institute, Seoul, Korea. ${ }^{4}$ Menzies School of Health Research and Charles Darwin University, Rocklands Drive, Casuarina, NT 0810, Australia.

Received: 4 April 2013 Accepted: 27 May 2013

Published: 31 May 2013

\section{References}

1. Obaro S, Greenwood B: Malaria and bacteraemia in African children. Lancet 2011, 378:1281-2.

2. Duggan MB, Beyer L: Enteric fever in young Yoruba children. Arch Dis Child 1975, 50:67-71.

3. Mabey DCW, Brown A, Greenwood BM: Plasmodium falciparum malaria and Salmonella infections in Gambian children. J Infect Dis 1987, 155:1319-1321.

4. Berkley J, Mwarumba S, Bramham K, Lowe B, Marsh K: Bacteremia complicating severe malaria in children. Trans R Soc Trop Med Hyg 1999, 93:283-286

5. Bronzan RN, Taylor TE, Mwenechanya J, Tembo M, Kayira K, Bwanaisa L, Njobvu A, Kondowe W, Chalira C, Walsh AL, Phiri A, Wilson LK, Molyneux ME, Graham SM: Bacteremia in Malawian children with severe malaria: prevalence, etiology, HIV coinfection, and outcome. J Infect Dis 2007, 195:895-904.

6. Graham SM, Walsh AL, Molyneux EM, Phiri A, Molyneux EM: Clinical presentation of non-typhoidal Salmonella bacteremia in Malawian children. Trans R Soc Trop Med Hyg 2000, 94:310-314

7. Scott JA, Berkley JB, Mwangi I, Ochola L, Uyoga S, Macharia A, Ndila C, Lowe BS, Mwarumba S, Bauni E, Marsh K, Williams TN: Relation between falciparum malaria and bacteraemia in Kenyan children: a population-based, case-control study and a longitudinal study. Lancet 2011, 378:1316-1323.

8. Alonso PL, Lindsay SW, Armstrong JR, Conteh M, Hill AG, David PH, Fegan G, de Francisco A, Hall AJ, Shenton FC, Greenwood BM, Conteh M, Cham K, Hill AG, David PH, Fegan G, Hall AJ: The effect of insecticide-treated bed nets on mortality of Gambian children. Lancet 1991, 337:1499-1502.
9. Kleinschmidt I, Schwabe C, Benavente L, Torrez M, Ridl FC, Segura JL, Ehmer $P$, Nchama GN: Marked increase in child survival after four years of intensive malaria control. AmJTrop Med Hyg 2009, 80:882-888.

10. Bookless A, Naftalin J: Typhoid fever complicated by benign tertian malaria. Br Med J 1945, 2:804-805

11. Piyaphanee $W$, Issarachaikul $R$, Soontarach $P$, Silachamroon U: Concurrent Salmonella bacteremia in P. vivax infection-a report of 2 cases at the Hospital for Tropical Diseases, Thailand. Southeast Asian J Trop Med Public Health 2007, 38:616-618.

12. Barber BE, William T, Grigg MJ, Menon J, Auburn S, Marfurt J, Anstey NM, Yeo TW: A prospective comparative study of knowlesi, falciparum, and vivax malaria in Sabah, Malaysia: high proportion with severe disease from Plasmodium knowlesi and Plasmodium vivax but no mortality with early referral and artesunate therapy. Clin Infect Dis 2013, 56:383-397.

13. Tan LK, Yacoub S, Scott S, Bhagani S, Jacobs M: Acute lung injury and other serious complications of Plasmodium vivax malaria. Lancet Infect Dis 2008, 8:449-454.

14. Kochar DK, Das A, Kochar SK, Saxena V, Sirohi P, Garg S, Kochar A, Khatri MP, Gupta V: Severe Plasmodium vivax malaria: a report on serial cases from Bikaner in northwestern India. AmJTrop Med Hyg 2009, 80:194-198.

15. Alexandre MA, Ferreira CO, Siqueira AM, Magalhães BL, Mourão MP, Lacerda MV, Alecrim M: Severe Plasmodium vivax malaria, Brazilian Amazon. Emerg Infect Dis 2010, 16:1611-1614.

16. Anstey NM, Douglas NM, Poespoprodjo JR, Price RN: Plasmodium vivax: clinical spectrum, risk factors and pathogenesis. Adv Parasitol 2012, 80:151-201.

17. Sur D, von Seidlein L, Manna B, Dutta S, Deb AK, Sarkar BL, Kanungo S, Deen JL, Ali M, Kim DR, Gupta VK, Ochiai RL, Tsuzuki A, Acosta CJ, Clemens JD, Bhattacharya SK: The malaria and typhoid fever burden in the slums of Kolkata, India: data from a prospective community-based study. Trans R Soc Trop Med Hyg 2006, 100:725-733.

18. Sur D, Ochiai RL, Bhattacharya SK, Ganguly NK, Ali M, Manna B, Dutta S, Donner A, Kanungo S, Park JK, Puri MK, Kim DR, Dutta D, Bhaduri B, Acosta CJ, Clemens JD: A cluster-randomized effectiveness trial of Vi typhoid vaccine in India. N Engl J Med 2009, 361:335-344.

19. Berkley J, Bejon P, Mwangi T, Gwer S, Maitland K, Williams TN, Mohammed S, Osier F, Kinyanjui S, Fegan G, Lowe BS, English M, Peshu N, Marsh K, Newton CR: HIV infection, malnutrition, and invasive bacterial infection among children with severe malaria. Clin Infect Dis 2009, 49:336-343.

20. White NJ: Determinants of relapse periodicity in Plasmodium vivax malaria. Malar J 2011, 10:297.

21. Price RN, Tjitra E, Guerra CA, Yeung S, White NJ, Anstey NM: Vivax malaria: neglected and not benign. AmJTrop Med Hyg 2007, 77(Suppl 6):79-87.

doi:10.1186/1475-2875-12-176

Cite this article as: Bhattacharya et al.: Vivax malaria and bacteraemia: prospective study in Kolkata, India. Malaria Journal 2013 12:176.

\section{Submit your next manuscript to BioMed Central and take full advantage of:}

- Convenient online submission

- Thorough peer review

- No space constraints or color figure charges

- Immediate publication on acceptance

- Inclusion in PubMed, CAS, Scopus and Google Scholar

- Research which is freely available for redistribution 